\title{
ACM Turing Award for 2020 Honors Alfred Vaino Aho and Jeffrey David Ullman
}

First about the Turing Award, informally known as the Nobel prize of computing. Namely, at the time of Alfred Nobel there were no computers and consequently there is no official Nobel prize for computing. However, since 1966 the ACM each year proclaims the most relevant computer award for life-time achievements.

The Turing Award was named for Alan Mathison Turing (1912-1954), the British mathematician who invented mathematical foundation and limits of computing, and who was a key contributor to the cryptanalysis of the Enigma cipher during World War II. Turing was often named "Albert Einstein of computer science" for the fundamental computing principles he invented or introduced. $\mathrm{He}$ is also the first to deal with the question if a Turing Machine can simulate true intelligence and found no principal reason why not. There were lots of discussions upon that issue, e.g. Roger Penrose's book "The Emperor's New Mind" in 1989 later resulting in Penrose-Hamerroff's thesis that it is quantum physics that enables human thinking stronger than computers.

Informatica and the author of this paper devoted lots of attention to Turing and the human-AI relations, e.g. in the 1995 special issue "Mind $<>$ Computer" edited by Matjaz Gams, Marcin Paprzycki, and Xindong Wu. In 2002, Minds and Machines published "The Turing Machine May Not Be the Universal Machine" by this author, based on a 2001 book "Weak Intelligence - the Principle and Paradox of Multiple Knowledge". The key to greater power of human thinking is attributed to multiple processes in the human brain that interact with each other during solution finding. However, attempts to formalize it were not fruitful.

In 2013, Informatica published a special issue "100 Years of Alan Turing and 20 Years of SLAIS" with special editors Dunja Mladenić, Stephen Muggleton, and Ivan Bratko (http://www.informatica.si/index.php/ informatica/issue/view/146/showToc). Also, an international multi-conference "Information society" (is.ijs.si) with several ties with the Informatica society, ACM Slovenia, SLAIS, and Informatika provides computer awards. Among them is the prestigious MichieTuring award (https://is.ijs.si/?page id=42) for life achievements, in memory of Alan Turing and his contemporary Donald Michie, who often visited the Informatica headquarters providing enjoyable discussions.

Back to the ACM 2020 award recipients: Alfred Vaino Aho, the Lawrence Gussman Professor Emeritus of Computer Science at Columbia University, and Jeffrey David Ullman, the Stanford W. Ascherman Professor Emeritus of Computer Science at Stanford University. Their major achievements were: introduction of the foundations of programming language compilers and algorithms, published in their highly influential books, which educated generations of computer scientists including the author of this text.

From the ACM proclamation (https://awards.acm.org/ about/2020-turing):

"The practice of computer programming and the development of increasingly advanced software systems underpin almost all of the technological transformations we have experienced in society over the last five decades," explains ACM President Gabriele Kotsis. "While countless researchers and practitioners have contributed to these technologies, the work of Aho and Ullman has been especially influential. They have helped us to understand the theoretical foundations of algorithms and to chart the course for research and practice in compilers and programming language design. Aho and Ullman have been thought leaders since the early 1970s, and their work has guided generations of programmers and researchers up to the present day."

"Aho and Ullman established bedrock ideas about algorithms, formal languages, compilers and databases, which were instrumental in the development of today's programming and software landscape," added Jeff Dean, Google Senior Fellow and SVP, Google AI. "They have also illustrated how these various disciplines are closely interconnected. Aho and Ullman introduced key technical concepts, including specific algorithms, that have been essential. In terms of computer science education, their textbooks have been the gold standard for training students, researchers, and practitioners."

Aho and Ullman co-authored nine influential books, including

- "The Design and Analysis of Computer Algorithms (1974)" by Aho, Ullman, and John Hopcroft

- "Principles of Compiler Design (1977)" by Aho and Ullman, often called the "Dragon Book".

Informatica and related societies join in celebrating the achievements of the ACM laureates that shaped the computer science and thus the human civilization.

Matjaž Gams

A note to the Informatica authors: In 2021 Informatica is introducing no. 5 on top of the classical 4 issues per year. The reason is based on a large amount of quality papers. No. 5 will be online-only, that means without paper printed journal copies, enabling fast on-line publications. 
